\title{
Coordinated Lot-sizing and Dynamic Pricing under a Supplier All-units Quantity Discount
}

Sandra Transchel, Department of Logistics, University of Mannheim, Email: sandra.transchel@bwl.uni-mannheim.de Stefan Minner, Faculty of Business, Economics and Statistics, University of Vienna, Email: stefan.minner@univie.ac.at

\begin{abstract}
We consider an economic order quantity model where the supplier offers an all-units quantity discount and a price sensitive customer demand. We compare a decentralized decision framework where selling price and replenishment policy are determined independently to simultaneous decision making. Constant and dynamic pricing are distinguished. We derive structural properties and develop algorithms that determine the optimal pricing and replenishment policy and show how quantity discounts not only influence the purchasing strategy but also the pricing policy. A sensitivity analysis indicates the impact of the fixed-holding cost ratio, the discount policy, and the customers' price sensitivity on the optimal decisions.
\end{abstract}

Keywords: Dynamic Pricing, Economic Order Quantity, Quantity-Discount, Procurement-Inventory Policies, Marketing-Operations Interface

\section{Introduction}

In recent years, the coordination of marketing and operations decisions has received a lot of attention. However, in practice these two decision areas are usually still independent. Even when the performance of marketing and operations is independently optimized in order to achieve their respective best operating level, it may lead to suboptimal performance for the firm as a whole (Ghose and Mukhopadhyay, 1993). In particular, the coordination of pricing strategies and operations decisions is still at an early stage and offers significant opportunities for improving supply chain performance. In this context, dynamic pricing strategies use intertemporal price discrimination to achieve a better match of supply with demand. On the supply side, the order quantity is a function of customer demand and fixed and variable purchasing costs. On the sales side, demand depends on the charged selling price. Therefore, determining the optimal dynamic selling prices and optimal order quantities is interdependent. It is common in practice that marketing determines the selling price first without taking into consideration overhead

\footnotetext{
* Corresponding author, Tel.: +49-621-181-1656, Fax: +49621-181-1653
}

purchasing or a supplier quantity discount. Then, given the selling price and the resulting demand forecast, operations decides on order quantities. However, due to the fact that marketing influences demand by the charged price and operations is responsible for satisfying this demand, price and procurement decisions are strongly connected and should be decided simultaneously. Fleischmann etal. (2004) review the linkage between pricing and operational decisions. They indicate different drivers for dynamic pricing strategies like promotions and other marketing-related instruments and more operations-driven pricing strategies known from supply chain coordination.

It is a common practice in that suppliers offer a discount for large order quantities. For this kind of price discrimination, a supplier designs a menu of price-quantity pairs and customers select their optimal purchasing volume. Dolan (1987) and Wilcox et al. (1987) provide several reasons for a firm to offer quantity discounts from both a marketing and an operations management point of view. If the supplier faces high setup costs which lead to a large lot-size and high holding costs, quantity discounts may reduce the inventory level immediately after stocking due to larger customer orders. Furthermore, suppliers offer quantity discounts 
for a better utilization of idle capacity in order to achieve economies of scale in manufacturing. From a marketing perspective, quantity discounts are used to stimulate sales, e.g., Neslin et al. (1995). From a financial point of view, the time value of money is taken into consideration. Because of the offered quantity discount, buyers decide to buy earlier and a larger quantity. Therefore, revenues are available earlier for possible reinvestment $(\mathrm{Be}-$ ranek, 1967).

In this paper we consider an economic order quantity (EOQ) model where the supplier offers an all-units quantity discount (AQD). This model is applicable to all goods that are purchased from a supplier that offers a quantity discount, e.g., durable consumer or industrial goods. We compare a decentralized decision framework where first marketing determines the optimal pricing strategy and then operations optimizes the replenishment policy to coordinated decision making where the retailer decides on pricing strategy and replenishment policy simultaneously. Hereby, we distinguish between two pricing strategies. In cases of constant pricing, the retailer determines the optimal selling price that is constant over an infinite planning horizon. In case of dynamic pricing the retailer varies the selling price over time. If price changes are free of charge, the optimal strategy is to change the price continuously over time (Rajan et al., 1992). If price changes are associated with costs, a continuous price adjustment is not beneficial. Therefore, there exists a finite optimal number of price changes. Netessine (2006) provides a variety of practical situations which cause that companies change prices only a limited number of times throughout the sales period. We analyze the benefits from coordinated dynamic pricing and replenishment compared to coordinated constant pricing and replenishment and to decentralized decision making. We assume that the number of price changes over an order cycle is given. However, in order to determine the optimal number of price changes, the results of Transchel and Minner (2005) can be used. They analyze a joint dynamic pricing and EOQ lot-sizing model without consideration of a supplier quantity discount and show that the optimal number of price changes and the optimal pricing and replenishment policy can be optimized by a two-stage program. That is, at the second stage, for a given number of price changes, the retailer determines the optimal pur- chasing and pricing strategy. At the first stage, the number of price changes is optimized anticipating the optimal purchasing and pricing strategy of the second stage. Transchel and Minner (2005) show how dynamic pricing can enhance operational efficiency by increasing the demand rate when inventories are high. The benefits of exploiting supply quantity discounts significantly depend on whether variable purchasing price reductions can offset additional holding costs from ordering minimum required quantities. Therefore it appears promising to use dynamic pricing here, too, to reduce the impact of holding costs. Furthermore, we extend Eliashberg and Steinberg (1993) who compare sequential and simultaneous optimization of lot-size and (constant) selling price without quantity discounts. They show that the optimal selling price in the case of simultaneous optimization is larger than in the case of sequential optimization which, in turn, results in a lower order frequency. This property does not necessarily hold if the supplier offers a quantity discount. We develop optimization models for three different decision frameworks: the decentralized framework where marketing and operations optimize independently, the coordinated-constant framework where the retailer optimizes a constant price and the order quantity simultaneously, and the coordinated-dynamic framework where the retailer employs a finite number of price changes over an order cycle. We provide analytical properties of the objective functions and present algorithms for determining the optimal replenishment policy and price strategy for the coordinated-constant and the coordinated-dynamic framework, respectively.

The remainder of the paper is organized as follows. After a literature review in Section 2, we introduce the models and the underlying assumptions and properties in Section 3. Section 4 compares the three frameworks for the special case of a linear price-response function. In a numerical example presented in Section 5 we show the benefit of coordinated decision making and in particular dynamic pricing compared to a decentralized decision framework. Furthermore, we show the impact of changing fixed costs, discount policy, and price sensitivity on the optimal decisions and the resulting benefit of coordination. Finally, we conclude the results, discuss limitation of the model, and indicate directions for further research in Section 6. 


\section{Literature review}

Several contributions analyze joint pricing and manufacturing situations. One of the earliest papers of integrated marketing/pricing and manufacturing decisions is by Whitin (1955) who shows that simultaneous decision-making on a constant price and ordering decision leads to significant profit increases compared to decentralized decisions. Eliashberg and Steinberg (1993) provide a comprehensive review of problems at the interface between marketing and operations. They compare differences between a decentralized and a coordinated optimization problem. In their overview, Bitran and Caldentey (2003) summarize dynamic pricing policies and their relation to revenue management. Elmaghraby and Keskinocak (2003) provide a review of literature and current practices in dynamic (intertemporal) pricing. Chan et al. (2004) review and classify papers according to a number of characteristics, e.g., stochastic vs. deterministic parameters and single vs. multiple products.

Benton and Park (1996) and Munson and Rosenblatt (1998) provide literature reviews on quantity discounts where they classify the literature under several discount schemes, different perspectives (buyer, supplier, joint), and other criteria like planning horizon and number of products. The impact of quantity discounts on the economic ordering decision has been investigated in several settings. In the literature, the most common discount policies are the all-units and the incremental-units discount policies. When the supplier offers an allunits quantity discount (AQD), the reduced purchasing price applies to the entire order quantity once the order quantity reaches a critical breakpoint. An incremental-units discount, however, only applies to all units in excess of a particular breakpoint. Hadley and Whitin (1963) develop a procedure for determining the optimal economic order quantity for both all-units and incremental units discount schemes. This approach is included in almost every textbook on operations management and assumes that the demand rate is known and constant over an infinite planning horizon and that the decision maker follows the objective to minimize average costs. Gupta (1988) provides an improved procedure for determining the optimal lot-size by consid- ering an upper bound for the relevant cost and Goyal and Gupta (1990) propose a further simplification which requires only a few EOQ calculations for determining the optimal lot-size. In Abad (1988a) and Abad (1988b) the simultaneous optimization of lot-size and selling price when the supplier offers an all-units or an incremental units discount is analyzed. He develops a procedure for determining the optimal lotsize and the optimal selling price. Burewell et al. (1991) extend the model of Abad (1988a) and allow for planned inventory shortages. They derive a similar procedure as Abad (1988a) to determine the optimal lot-size and selling price for two classes of demand functions, iso-elastic and linear. More recently, discount pricing schedules have received growing attention to improve the coordination between vendors and buyers, see e.g., Weng (1995), Rubin and Benton (2003), and Wang (2005).

Inventory problems with simultaneous optimization of the replenishment policy and a dynamic pricing strategy where only a limited number of price changes is allowed has been investigated less frequently. The main motivation for considering a limited number of price changes are organizational costs associated with each price change. Abad (1997) formulates a model where the reseller responds to a temporary price reduction of the supplier by an adjustment of the retailer's own selling price. Abad (1997) considers that the reseller is allowed to charge two selling prices in each order cycle. The presented model optimizes the first (discounted) selling price and fixes the second selling price to the optimal constant price. He presents a procedure where a temporarily reduced selling price yields a higher cycle profit than in the case of a static selling price. As shown in Transchel and Minner (2005), the majority of benefits from dynamic pricing can be captured by very few different price levels and, therefore, a discrete number of changes balances benefits and costs of price changes. Netessine (2006) analyzes a pricing problem with a limited number of price changes in a dynamic, deterministic environment and a capacity constraint. $\mathrm{He}$ characterizes the impact of capacity constraints on the optimal prices and the timing of price changes and provides several comparative statics results. 


\section{Model}

\subsection{Assumptions}

Consider a monopolistic retailer who is selling a product on a single market over an infinite planning horizon. We assume that customer demand follows a function of the selling price $P$ and arrives continuously at a rate of $D(P)$ which is a differentiable and strictly non-increasing function in $P$ with $D(P) \geq 0$ and $D^{\prime}(P)<0$. Furthermore, let $\varepsilon$ define the price elasticity of $D(P)$ with $\varepsilon=-\frac{D^{\prime}(P)}{D(P)} P$ which is the percentage change in demand in response to a percentage change in price. Based on the price elasticity, we define a class of price-response functions as follows:

Defintion 1 A price-response function $D(P)$ has an increasing price elasticity (IPE), if $\frac{\partial \varepsilon}{\partial P} \geq 0$.

The intuition behind the IPE property is that with a price increase by a certain percentage demand decreases by a larger percentage. At every point in time, the demand rate depends only on the current price, i.e., customers are willing to buy as soon as the price is below their individual reservation price. Let $\bar{P}$ denote the market reservation price with $\bar{P} \leq \infty$. We assume that by charging a reduced selling price at the beginning of an order cycle customers do not behave strategically and buy in expectation of future prices (forward buying and postponement).

We follow the assumptions of the EOQ model. With the release of any single order there is an associated setup cost $F$. Furthermore, the supplier has no capacity constraints and the overall order quantity is delivered in one shipment without any delay. Products delivered but not yet sold are kept in inventory subject to holding cost per unit and unit of time. The replenishment orders are placed in batches of size $Q$ every $T$ periods over an infinite planning horizon. Backorders are not permitted. The supplier offers a regular purchasing price $c_{0}$ per unit and an AQD schedule with $l=0, \cdots, L$ different purchasing price categories where the discount is $r_{l}$ percent per unit if the order quantity is larger than or equal to a breakpoint quantity $\bar{Q}_{l}$. The AQD policy with multiple breakpoints is characterized by a vector

$$
\begin{aligned}
& \left\{\left(r_{0}, \bar{Q}_{0}\right),\left(r_{1}, \bar{Q}_{1}\right), \cdots,\left(r_{L}, \bar{Q}_{L}\right)\right. \\
& \left.\quad \mid r_{0}<r_{1}<\cdots<r_{L}, \bar{Q}_{0}<\bar{Q}_{1}<\cdots<\bar{Q}_{L}\right\}
\end{aligned}
$$

with $r_{0}=\bar{Q}_{0}=0$. Let $c_{l}:=\left(1-r_{l}\right) c_{0}$ be the reduced purchase price for a unit if the order quantity $Q \in\left[\bar{Q}_{l}, \bar{Q}_{l+1}\right)$ with $c_{0}>c_{1}>\cdots>c_{L}$ and $\bar{Q}_{L+1}=\infty$. Inventory holding costs depend, among others, on the cost of capital that, in turn, depends on the purchase price $c_{l}$ and are denoted by $h_{l}$ per unit and unit of time. We assume that $h_{l}$ is an increasing function of $c_{l}$.

\subsection{Decentralized decisions}

Assume that the selling price and the purchasing strategy are determined by separated decisionmaking units. First, marketing optimizes the selling price and generates customer demand. Then, given this demand forecast, operations determines the optimal replenishment policy taking into account the supplier's quantity discount. Marketing does not take into account fixed ordering costs and does not anticipate purchasing price discounts as a result of order quantities because the discount that is actually applied is unknown until the operations decision is taken. Marketing's objective function is as follows:

$$
\tilde{\Pi}(P)=\left(P-c_{0}\right) D(P) .
$$

The optimal selling price $\tilde{P}^{*}$ is obtained from the first-order condition $P+\frac{D(P)}{D^{\prime}(P)}=c_{0}$. Given the demand forecast $\tilde{D}^{*}=D\left(\tilde{P}^{*}\right)$, operations minimizes average costs of replenishment and inventory taking into account the supplier quantity discount. Hadley and Whitin (1963) developed a two-stage algorithm in order to determine the optimal replenishment policy. A first stage iteratively calculates the constrained economic order quantity $\tilde{Q}_{l}^{*}$ and the resulting costs starting from the highest discount $c_{L}$ until the first index $l_{0}$ is found where the solution satisfies $\tilde{Q}_{l_{0}}^{*} \geq \bar{Q}_{l_{0}}$ and $\tilde{Q}_{l}^{*}<\bar{Q}_{l}$ for all $l>l_{0}$. Thus,

$$
\begin{aligned}
& \tilde{Q}_{l_{0}}^{*}=\sqrt{\frac{2 F D\left(\tilde{P}^{*}\right)}{h_{l_{0}}}} \geq \bar{Q}_{l_{0}} \text { and } \\
& \tilde{C}_{l_{0}}^{*}=\sqrt{2 F h_{l_{0}} D\left(\tilde{P}^{*}\right)}
\end{aligned}
$$

At a second stage, the cost of this inner solution $\tilde{C}_{l_{0}}^{*}$ is compared with the costs of all breakpoint quantities larger than $\tilde{Q}_{l_{0}}^{*}$, i.e., $\tilde{C}_{l}\left(\bar{Q}_{l}\right)$ for $l=l_{0}+1, \ldots, L$ with

$$
\tilde{C}_{l}\left(\bar{Q}_{l}\right)=c_{l} D\left(\tilde{P}^{*}\right)+\frac{h_{l}}{2} \bar{Q}_{l}+F \frac{D\left(\tilde{P}^{*}\right)}{\bar{Q}_{l}} .
$$


Thus, the optimal profit in the case of decentralized decision making is as follows:

$$
\begin{aligned}
\tilde{\Pi}^{*}= & \left(\tilde{P}^{*}-c_{0}\right) D\left(\tilde{P}^{*}\right) \\
& -\min \left\{\tilde{C}_{l_{0}}^{*}, \tilde{C}_{l}\left(\bar{Q}_{l}\right) \mid l=l_{0+1}, \ldots, L\right\} .
\end{aligned}
$$

\subsection{Coordinated-constant decision}

In case of coordinated decision making, we simultaneously optimize lot-size $Q$ and selling price $P$. The optimization problem for a particular purchasing price $c_{l}$ is given by

$$
\begin{aligned}
\Pi_{l}^{*}(P, Q)= & \max _{P, Q}\left(\left(P-c_{l}\right) D(P)\right. \\
& \left.-\frac{h_{l}}{2} Q-F \frac{D(P)}{Q}\right)
\end{aligned}
$$

$$
\text { s.t. } Q \geq \bar{Q}_{l} .
$$

A relaxation of (4) and differentiating (3) with respect to $Q$ and $P$ yields the necessary first-order conditions for an inner solution (e.g., see Whitin (1955) and Eliashberg and Steinberg (1993))

$$
Q_{l}^{*}=\sqrt{\frac{2 F D\left(P_{l}^{*}\right)}{h_{l}}} \text { and } P_{l}^{*}+\frac{D\left(P_{l}^{*}\right)}{D^{\prime}\left(P_{l}^{*}\right)}=c_{l}+\frac{F}{Q_{l}^{*}}
$$

where the optimal selling price $P_{l}^{*}$, conditional that the purchasing price is $c_{l}$, is represented as an implicit function of the optimal order quantity. For a particular purchasing price $c_{l}$ the optimal order quantity is only feasible if $\bar{Q}_{l} \leq Q_{l}^{*}$. Given this constraint, a transformation of $Q_{l}^{*}$ from (5) yields that an inner solution $P_{l}^{*}$ is only feasible if $P_{l}^{*}$ is lower than a break price $\bar{P}_{l}$ for a given unit purchasing cost $c_{l}$ :

$$
P_{l}^{*} \leq D^{-1}\left(\frac{h_{l} \bar{Q}_{l}^{2}}{2 F}\right)=: \bar{P}_{l}
$$

where $D^{-1}($.$) denotes the inverse of the price-$ response function. The inverse function does exist due to the fact that $D(P)$ is a strictly monotone function. Using this result, the AQD policy can be characterized by

$$
\begin{aligned}
& \left\{\left(r_{0}, \bar{P}_{0}\right),\left(r_{1}, \bar{P}_{1}\right), \cdots,\left(r_{L}, \bar{P}_{L}\right)\right. \\
& \left.\quad \mid r_{0}<r_{1}<\cdots<r_{L}, \bar{P}_{0}>\bar{P}_{1}>\cdots>\bar{P}_{L}\right\}
\end{aligned}
$$

where $\bar{P}_{0}=\bar{P}$. Substituting the optimal order quantity into (3), the two-variable problem is reduced to a single-variable problem that only depends on $P$ :

(7) $\Pi_{l}(P)=\left(P-c_{l}\right) D(P)-\sqrt{2 F h_{l} D(P)}$,

(8) $\quad$ s.t. $P \leq \bar{P}_{l}$.

\section{Properties of an optimal pricing and lot- sizing policy}

The following properties characterize the profit function (7).

Property 1 For $P \geq 0$, (7) is either a concaveconvex function or a strictly concave function of $P$ with $\lim _{P \rightarrow 0} \Pi_{l}(P)<0$ and $\lim _{P \rightarrow \bar{P}} \Pi_{l}(P)=0$.

The proof of Property 1 is given in Appendix A.1. Abad (1988a) reduces (3) to a single-variable problem that only depends on the order quantity $Q$. He shows that if the first-order condition with respect to $P$ yields a closed-form solution $P^{*}(Q)$, as it is the case for linear and iso-elastic price-response functions, the reduced profit function is a convexconcave function of $Q$ and develops a procedure to determine the optimal price and lot-size. Based on this result, Abad (1988a) gives an algorithm to determine the overall optimal lot-size. Note that we do not need the required condition of having a closed-form solution for $P^{*}(Q)$.

Property 2 For an arbitrary fixed selling price $P,(7)$ is a strictly decreasing function of $c_{l}$. Therefore, the profit functions $\Pi_{l}$ and $\Pi_{l^{\prime}}$ for different unit purchasing costs $c_{l}$ and $c_{l^{\prime}}$ do not intersect and $\Pi_{l}(P)>\Pi_{l^{\prime}}(P)$ for all $P$ and $c_{l}<c_{l^{\prime}}$.

From the partial derivative of (7) with respect to $c_{l}$ and the assumption that $h_{l}$ increases in $c_{l}$, it is easy to verify that $\frac{\partial \Pi_{l}}{\partial c_{l}}<0$. Implicitly, it follows that the profit function $\Pi_{l}(P)>\Pi_{l^{\prime}}(P)$ for $c_{l}<c_{l^{\prime}}$.

Theorem 1 Let $l_{0}$ be the largest index of a discount where the local optimum $Q_{l_{0}}^{*}$ is feasible, i.e., $\bar{Q}_{l_{0}} \leq Q_{l_{0}}^{*}$ and $\bar{Q}_{l}>Q_{l}^{*}$ for all $l=l_{0}+1, \cdots, L$. Then for all $l<l_{0}, \Pi_{l}(P)<\Pi_{l_{0}}\left(P_{l_{0}}^{*}\right)$ for all $P$.

The proof follows directly from Properties 1 and 2 with the implication that all discounts that are lower than the discount $r_{l_{0}}$ can be omitted for the determination of the optimal solution. If $\Pi_{l}\left(P_{l}^{*}\right)<0$ for all $c_{l}$, then $Q^{*}=0$. To find the optimal value of $P$, we have to find the profit maximizing price in each interval $\left(\bar{P}_{l+1}, \bar{P}_{l}\right]$ and compare these profits to determine the global optimum.

For the following illustration we assume that the supplier offers a single price break $\bar{Q}_{1}$. Then, there are 3 cases for the optimal price $P^{*}$.

1. The free local optimum $P_{1}^{*}$ for the reduced purchasing price $c_{1}$ is a feasible solution, i.e., $P_{1}^{*} \leq \bar{P}_{1}$. 
Figure 1: Average Profit Curve Case 1

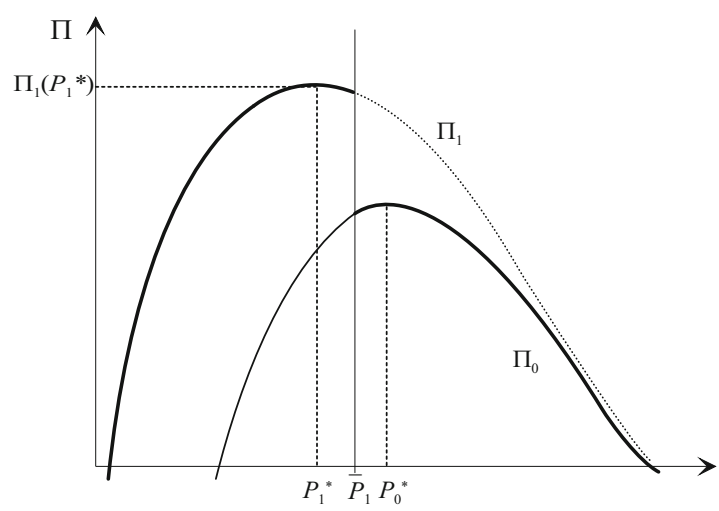

Figure 2: Average Profit Curve Case 2

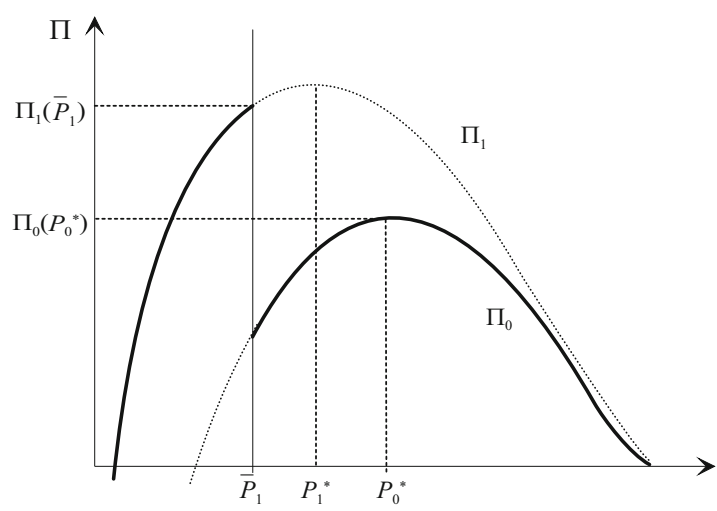

2. The free local optimum $P_{1}^{*}$ is infeasible and the breakpoint profit (the retailer orders the breakpoint quantity $\bar{Q}_{1}$ at the reduced purchasing price $c_{1}$ ) is larger than the optimal profit given the regular purchasing price $c_{0}$, $\Pi_{1}\left(\overline{P_{1}}\right)>\Pi_{0}\left(P_{0}^{*}\right)$.

3. The free local optimum $P_{1}^{*}$ is infeasible and the profit where the retailer orders the breakpoint quantity is lower than the optimal profit for the regular purchasing price, $\Pi_{1}\left(\bar{P}_{1}\right)<\Pi_{0}\left(P_{0}^{*}\right)$.

In Case 1, as shown in Figure 1, the breakpoint price $\bar{P}_{1}$ is large enough such that the optimal selling price $P_{1}^{*}$ is a feasible solution. Figures 2 and 3 illustrate Cases 2 and 3 where $P_{1}^{*}$ is an infeasible solution. In Case 2 the breakpoint profit is larger than the optimal profit for the regular purchasing price $c_{0}$ and in Case 3 the opposite holds. If $\Pi_{1}\left(\bar{P}_{1}\right)=\Pi_{0}\left(P_{0}^{*}\right)$, the retailer is indifferent between ordering $\bar{Q}_{1}$ or $Q_{0}^{*}$. The bold lines represent the feasible profit curves. Properties 1 and 2 and Theorem 1 allow the following algorithm for calculating the optimal selling price.
Figure 3: Average Profit Curve Case 3

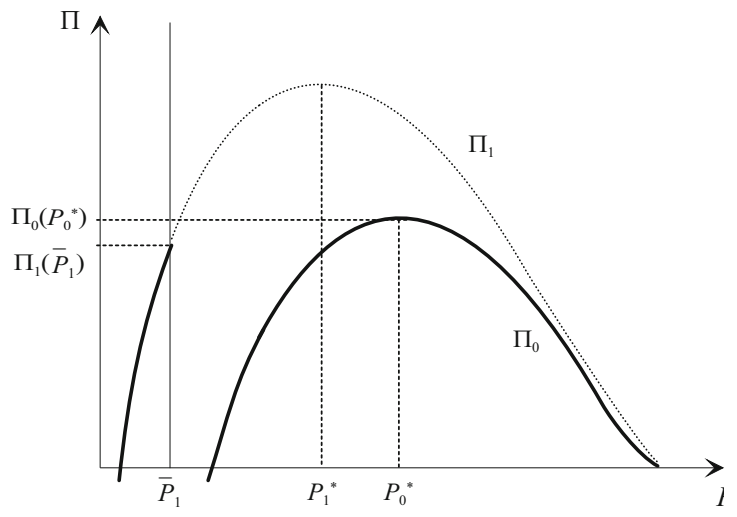

\section{Algorithm 1 for determining the optimal price and lot-size}

Set $l:=L$,

\section{REPEAT}

Calculate the optimal selling price $P_{l}^{*}$ by solving the first-order condition $\frac{\partial \Pi_{l}}{\partial P} \stackrel{!}{=} 0$,

$$
\frac{D(P)}{D^{\prime}(P)}+P-c_{l}-\sqrt{\frac{F h_{l}}{2 D(P)}}=0,
$$

calculate $\Pi_{l}^{*}:=\Pi_{l}\left(\bar{P}_{l}\right)$,

$$
l:=l-1 \text {, }
$$

UNTIL $P_{l}^{*}<\bar{P}_{l}$.

(The loop stops at the index $l_{0}$, the largest index for which $P_{l_{0}}^{*} \leq \bar{P}_{l_{0}}$, i.e., the local optimum $P_{l_{0}}^{*}$ is an inner solution.)

Calculate $\Pi_{l_{0}}^{*}:=\Pi_{l_{0}}\left(P_{l_{0}}^{*}\right)$.

The optimal profit is determined from

$$
\Pi^{*}=\left\{\begin{array}{c}
\max \left\{\Pi_{l}^{*} \mid l \in\left\{l_{0}, \cdots, L\right\}\right\} \\
\text { if at least one } \Pi_{l}^{*} \geq 0 \\
0 \quad \text { otherwise }
\end{array}\right.
$$

Let $l^{*}:=\operatorname{argmax}\left\{\Pi_{l}^{*}\right\}$. Then the optimal price and the optimal order quantity are given by

$$
P^{*}=\left\{\begin{array}{ll}
\left.\operatorname{argmax}\left\{\Pi_{l^{*}}^{*}(P)\right\}\right\} & \text { if } \Pi^{*} \geq 0 \\
\bar{P} & \text { otherwise }
\end{array},\right.
$$

and

$$
Q^{*}=\left\{\begin{array}{ll}
\sqrt{\frac{2 F D\left(P^{*}\right)}{h_{l^{*}}},}, & \text { if } P^{*}=P_{l_{0}}^{*} \\
\bar{Q}_{l}, & \text { if } P^{*}=\bar{P}_{l}
\end{array} .\right.
$$

END 
The important property for this algorithm to work is that the profit curves do not intersect which gives that the profit function for a discount rate $r_{l}$ is located above of profit functions for discount rates $r_{l^{\prime}}$ with $l^{\prime}<l$.

\section{Impact of coordinated decision making on price and purchasing policy}

Now we compare decentralized (sequential) and coordinated (simultaneous) decision making on pricing and purchasing strategy. Eliashberg and Steinberg (1993) analyze the sequential and coordinated decision-making problem without a supplier quantity discount. They show that decentralized decision making, where marketing first decides on the selling price without taking into account operation's overhead costs and then operations determines the optimal purchasing policy, leads to an underestimated selling price and an overestimated demand rate. This, in turn, leads to larger lot-sizes than in case of coordinated decision making. However, if the supplier offers a quantity discount, these effects do not necessarily hold anymore. For a particular discount rate $r_{l}$, a comparison of the first-order condition of decentralized and coordinated decision making gives

$$
\text { (9) } \begin{aligned}
& \frac{D(\tilde{P})}{D^{\prime}(\tilde{P})}+\tilde{P}=c_{0} \quad \text { and } \\
& \frac{D(P)}{D^{\prime}(P)}+P=c_{l}+\frac{F}{Q} \text { for all } l=0, \cdots, L .
\end{aligned}
$$

Under decentralized decision making, the optimal selling price $\tilde{P}^{*}$ is only based on the regular purchasing costs $c_{0}$. However, in the coordinated case, the price $P^{*}$ takes into account both overhead costs and an eventually used quantity discount.

Property 3 If $D(P)$ has an increasing price elasticity and the retailer does not use a quantity discount $(l=0)$, then the optimal selling price in case of decentralized decision making is lower than under simultaneous optimization, i.e., $\tilde{P}^{*}<P^{*}$ and $D\left(\tilde{P}^{*}\right)>D\left(P^{*}\right)$. If $l>0$, the coordinated case yields a higher price in comparison to the decentralized system if the optimal coordinated selling price satisfies $P_{l}^{*}>D^{-1}\left(\frac{F h_{l}}{2\left(c_{0}-c_{l}\right)^{2}}\right)$.

The proof is given in Appendix A.2. The direction of the price change is influenced by two effects: the overhead cost effect and the discount effect. The overhead cost effect describes the impact of coor- dinated decision making. In the case of sequential decision making, marketing does not take into account that operations uses a quantity discount. Depending on the actually paid purchasing costs, marketing either underestimates or overestimates operations costs that additionally contain setup cost and holding costs. The discount effect leads to a decreasing selling price. Due to the increasing lot-size by using the supplier quantity discount, the retailer decreases the prices in order to increase the demand and reduce holding costs. Whether the optimal selling price increase or decrease compared to the decentralized case depends on which effect dominates.

\subsection{Coordinated-dynamic decision}

Let us assume that the retailer can change the selling price $N$ times at time $t_{i}, i=0, \cdots, N-1$ within each order cycle where each price change is subject to administrative costs. $\kappa(N)$ defines a non-decreasing function of $N$. At time $t_{0}=0$, the retailer places the order and sets the selling price $P_{1}$ which holds until $t_{1}$. At time $t_{1}$, the retailer changes the price to $P_{2}$. We assume that over a time interval $\left[t_{i-1}, t_{i}\right)$, the retailer charges a price $P_{i}$. Let $t_{N}$ denote the cycle length. The objective is to optimize the purchasing and dynamic pricing strategy which includes the determination of the optimal prices and the optimal times where the price is adjusted. This problem can be solved by a two-stage optimization approach: At the second stage, for a given $N$, the retailer determines the optimal purchasing and pricing strategy. At the first stage, the optimal number of price changes $N$ is optimized anticipating the optimal purchasing and pricing strategy of the second stage. For a simplified notation, let $D_{i}=D\left(P_{i}\right)$. The two-stage problem can be formulated as follows:

$$
\Pi^{*}=\max _{N}\left[\max _{l, \mathbf{P}_{N}, \mathbf{t}_{N}}\left\{\Pi_{l}^{(N)}-\kappa(N)\right\}\right],
$$

with

$$
\begin{aligned}
& \Pi_{l}^{(N)}=\frac{1}{t_{N}}\left[\sum_{i=1}^{N}\left(P_{i}-c_{l}\right) D_{i}\left(t_{i}-t_{i-1}\right)\right. \\
& -\frac{h_{l}}{2} \sum_{i=1}^{N} D_{i}\left(t_{i}-t_{i-1}\right)^{2} \\
& \left.-h_{l} \sum_{i=1}^{N-1}\left(\left(t_{i}-t_{i-1}\right) \sum_{j=i+1}^{N} D_{j}\left(t_{j}-t_{j-1}\right)\right)-F\right] .
\end{aligned}
$$


(12)

$$
\begin{array}{ll}
\text { s.t. } & P_{i} \leq \bar{P} \quad \forall i=1, \cdots, N \\
& \sum_{i=1}^{N} D_{i}\left(t_{i}-t_{i-1}\right) \geq \bar{Q}_{l} .
\end{array}
$$

Equation (11) represents the average retailer profit given $c_{l}$, a price vector $\mathbf{P}_{N}=\left(P_{1}, \cdots, P_{N}\right)$ and a timing vector $\mathbf{t}_{N}=\left(t_{1}, \cdots, t_{N}\right)$. The first term is the unit revenue minus direct purchasing cost (when the retailer uses the discount rate $r_{l}$ ) for each interval multiplied by the respective demand rate and the length of the interval. In the second and third term we subtract inventory holding costs. These consist (see Figure 5) of the average of initial and final inventory in each interval (triangles) and the inventory that has to be carried over the entire interval $\left[t_{i}-t_{i-1}\right]$ to cover the demand of all subsequent intervals $j=i+1, \cdots, N$ with an amount of $\sum_{j=i+1}^{N} D_{j}\left(t_{j}-t_{j-1}\right)$ (rectangles). Constraint (12) ensures that the selling price does not exceed the reservation price and by constraint (13) the lot-size must be larger than or equal to the breakpoint $\bar{Q}_{l}$ to ensure that the used unit price is attained.

A detailed analysis of how to solve (11) is given in Transchel and Minner (2005). The order quantity can be determined as the sum over all intervals, demand rates multiplied by the interval length and therefore implicitly by the price decisions in all subintervals of an order cycle

$$
Q=\sum_{i=1}^{N} D_{i}\left(t_{i}-t_{i-1}\right)
$$

\section{Properties of an optimal pricing and lot- sizing policy}

By algebraic manipulations (see Appendix A.3), we can simplify (11) to

$$
\begin{aligned}
\Pi_{l}^{(N)}= & \frac{1}{t_{N}}\left[\sum_{i=1}^{N}\left(P_{i}-c_{l}-\frac{h_{l}}{2}\left(t_{i}+t_{i-1}\right)\right)\right. \\
& \left.\times D_{i}\left(t_{i}-t_{i-1}\right)-F\right] .
\end{aligned}
$$

In order to maximize the average profit, we differentiate (15) with respect to $P_{i}$ and $t_{i}, i=1, \cdots, N$. The necessary first-order conditions with respect to $P_{i}$ give

$$
\text { (16) } \begin{aligned}
& \frac{\partial \Pi_{l}^{(N)}}{\partial P_{i}}=\frac{1}{t_{N}}\left[D_{i}\left(t_{i}-t_{i-1}\right)\right. \\
& \left.+\left(P_{i}-c_{l}-\frac{h_{l}}{2}\left(t_{i}+t_{i-1}\right)\right) D_{i}^{\prime}\left(t_{i}-t_{i-1}\right)\right] \stackrel{!}{=} 0, \\
\Leftrightarrow & \frac{D_{i}}{D_{i}^{\prime}}+P_{i} \stackrel{!}{=} c_{l}+\frac{h_{l}}{2}\left(t_{i}+t_{i-1}\right) .
\end{aligned}
$$

The optimal price $P_{i}^{*}$ in a particular time interval $\left[t_{i-1}, t_{i}\right)$ for $i=1, \cdots, N$ must satisfy (16) and depends on the initial and final point of this interval. For price-response functions with a price-elasticity that is non-decreasing in price, the optimal selling price $P_{i}^{*}$ is non-decreasing in $i$ (see Transchel and Minner (2005)). Figures 4 and 5 illustrate the optimal pricing policy and the corresponding inventory development for a certain $N$ and $c_{l}$. The first-order condition of $\Pi_{l}^{(N)}$ with respect to $t_{i}$ for $i=1, \ldots, N-1$ gives

$$
\begin{aligned}
& \frac{\partial \Pi_{l}^{(N)}}{\partial t_{i}}=\frac{1}{t_{N}}\left[\left(P_{i}-c_{l}\right) D_{i}\right. \\
& \left.\quad-\left(P_{i+1}-c_{l}\right) D_{i+1}-h_{l} t_{i}\left(D_{i}-D_{i+1}\right)\right] \stackrel{!}{=} 0 .
\end{aligned}
$$

Figure 4: Illustration of the pricing policy

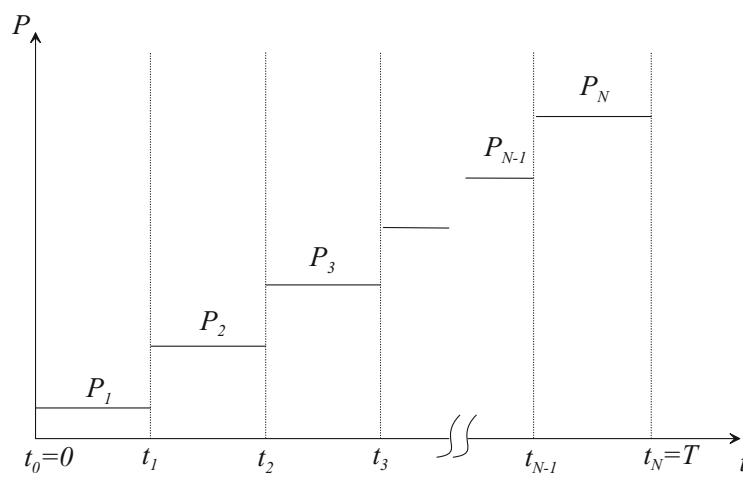

Figure 5: Illustration of inventory level

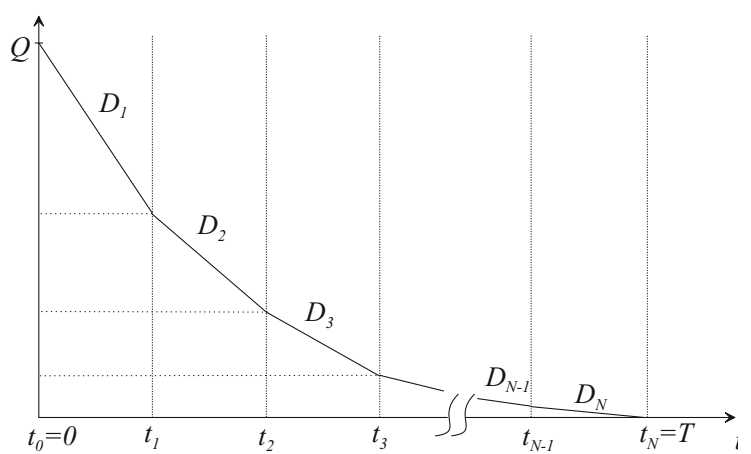


Therefore, the optimal times $t_{i}^{*}$ must satisfy

$$
\begin{aligned}
t_{i}^{*}= & \frac{\left(P_{i}^{*}-c_{l}\right) D_{i}-\left(P_{i+1}^{*}-c_{l}\right) D_{i+1}^{*}}{h_{l}\left(D_{i}^{*}-D_{i+1}^{*}\right)} \\
& i=1, \cdots, N-1 .
\end{aligned}
$$

From (17) we find that the optimal time $t_{i}^{*}$ is a function of the optimal price $P_{i}^{*}$ of the current interval, the optimal price $P_{i+1}^{*}$ of the next interval, and the corresponding optimal demand rates. Given the results of (16) and (17), it is apparent that $t_{i}^{*}$ can be represented as a function of its predecessor $t_{i-1}^{*}$ and its successor $t_{i+1}^{*}$. With the initial condition $t_{0}=0$ it follows that $t_{1}^{*}$ is only a function of its successor $t_{2}^{*}$. Furthermore, $t_{2}^{*}$ is a function of $t_{1}^{*}$ and $t_{3}^{*}$. By substituting $t_{1}^{*}$ as a function of $t_{2}^{*}$ and some algebraic manipulations, the time $t_{2}^{*}$ can be represented as a single-variable function of $t_{3}^{*}$. An iterative transformation and substitution gives that from $\left[t_{1}^{*}\left(t_{2}\right), t_{2}^{*}\left(t_{1}, t_{3}\right), \cdots, t_{N}^{*}\left(t_{N-1}\right)\right]$ follows $\left[t_{1}^{*}\left(t_{2}\right), t_{2}^{*}\left(t_{3}\right), \cdots, t_{N-1}^{*}\left(t_{N}\right)\right]$. Now, by backward insertion, each optimal time where the selling price is changed can be represented as a function $t_{i}^{*}\left(t_{N}\right)$ of the cycle length. Therefore, the optimal prices and the optimal demand rates can be reduced to functions that only depend on the cycle length $t_{N}$, e.g., $\left(P_{i}^{*}\left(t_{N}\right), D_{i}^{*}\left(t_{N}\right)\right)$, and the maximization problem reduces to the following single-variable problem:

$$
\begin{aligned}
& \Pi_{l}^{(N)}\left(t_{N}\right):=\Pi_{l}^{(N)}\left(P_{1}^{*}\left(t_{N}\right), \cdots,\right. \\
& \left.P_{N}^{*}\left(t_{N}\right), t_{1}^{*}\left(t_{N}\right), \cdots, t_{N-1}^{*}\left(t_{N}\right), t_{N}\right) \\
= & \frac{1}{t_{N}}\left[\sum_{i=1}^{N}\left(P_{i}^{*}\left(t_{N}\right)-c_{l}-\frac{h_{l}}{2}\left(t_{i}^{*}\left(t_{N}\right)+t_{i-1}^{*}\left(t_{N}\right)\right)\right)\right. \\
\times & \left.D_{i}^{*}\left(t_{N}\right)\left(t_{i}^{*}\left(t_{N}\right)-t_{i-1}^{*}\left(t_{N}\right)\right)-F\right] .
\end{aligned}
$$

In Section 4, we will show that for a linear priceresponse function this iterative procedure yields closed-form expression for $P_{i}^{*}\left(t_{N}\right)$ for all $i=1, \cdots, N$. In the general case, the dependencies have to be solved numerically. As for the static pricing problem the profit function $\Pi_{l}^{(N)}\left(t_{N}\right)$ has to possess some particular characteristics that enable to find the optimal solution efficiently.

Conjecture 1 For an arbitrary feasible $t_{N}(0 \leq$ $\left.P_{i}^{*}\left(t_{N}\right) \leq \bar{P}, \forall i=1, \cdots, N\right)$, (18) is a decreasing function of $c_{l}$. Therefore, if the profit function is concave, quasi-concave, or concave-convex, then $\Pi_{l}^{(N)}\left(t_{N}\right)$ and $\Pi_{l^{\prime}}^{(N)}\left(t_{N}\right)$ do not intersect for $c_{l} \neq c_{l^{\prime}}$, i.e., $\Pi_{l}^{(N)}\left(t_{N}\right)>\Pi_{l-1}^{(N)}\left(t_{N}\right)$ for all $l=1, \cdots, N-1$.
For general price-response functions the proof of Conjecture 1 is analytically intractable. However, in the next section we show for linear price-response functions that Conjecture 1 holds. The shape of (18) depends on the price-response function. If $D(P)$ is such that (18) is concave, quasi-concave, or concave-convex, similar to the constant pricing case, for a fixed $c_{l}$, the following algorithm can be applied to determine the optimal solution. Otherwise a complete enumeration over all inner solutions and breakpoints has to be used in order to determine the optimal solution. Similar to the results under static pricing, a simple algorithm can be used to determine the optimal cycle $t_{N}$ for any $c_{l}$.

\section{Algorithm 2 to determine the optimal price strategy and lot-size}

Set $l:=L$,

\section{REPEAT}

Solve the constrained optimization problem:

(19) $\max _{t_{N}} \quad\left\{\Pi_{l}^{(N)}\left(t_{N}\right)\right\}$

(20) $\quad$ s.t. $\quad P_{i}\left(t_{N}\right) \leq \bar{P} \quad \forall i=1, \cdots, N$.

$l:=l-1$

UNTIL $\sum_{i=1}^{N} D_{i}^{*}\left(t_{N}^{*}\right)\left(t_{i}^{*}\left(t_{N}\right)-t_{i-1}^{*}\left(t_{N}\right)\right) \geq \bar{Q}_{l+1}$.

Let $l_{0}$ be the largest index for which the free optimum $Q_{l_{0}}^{*}=\sum_{i=1}^{N} D_{i}^{*}\left(t_{N}^{*}\right)\left(t_{i}^{*}\left(t_{N}^{*}\right)-t_{i-1}^{*}\left(t_{N}^{*}\right)\right)$ is a feasible solution, i.e., $Q_{l_{0}}^{*} \geq \bar{Q}_{l_{0}}$. Calculate the optimal profit

$$
\begin{aligned}
\Pi_{l_{0}}^{(N)^{*}}:= & \Pi_{l_{0}}^{(N)}\left[P_{1}^{*}\left(t_{N}^{*}\right), \cdots,\right. \\
& \left.P_{N}^{*}\left(t_{N}^{*}\right), t_{1}^{*}\left(t_{N}^{*}\right), \cdots, t_{N-1}^{*}\left(t_{N}^{*}\right), t_{N}^{*}\right]
\end{aligned}
$$

and the breakpoint profits

$$
\begin{aligned}
\Pi_{l}^{(N)^{*}}:=\Pi_{l}^{(N)}\left[P_{1}^{*}\left(t_{N_{l}}\right), \cdots,\right. \\
\left.\quad P_{N}^{*}\left(t_{N_{l}}\right), t_{1}^{*}\left(t_{N_{l}}\right), \cdots, t_{N-1}^{*}\left(t_{N_{l}}\right), t_{N_{l}}\right]
\end{aligned}
$$

for all $l$ from $l_{0}+1$ to $L$ where $t_{N_{l}}$ denotes the optimal cycle length given that the retailer orders the breakpoint quantity $\bar{Q}_{l}$.

The optimal profit is determined from

$$
\Pi^{(N)^{*}}=\left\{\begin{array}{cl}
\max \left\{\Pi_{l}^{(N)^{*}} \mid l \in\left\{l_{0}, \cdots, L\right\}\right\} & \text { if at least one } \Pi_{l}^{(N)^{*}} \geq 0 . \\
0 \quad & \text { otherwise }
\end{array}\right.
$$


Let $l^{*}=\operatorname{argmax}\left\{\Pi_{l}^{(N)^{*}}\right\}$. For the case $\Pi^{(N)^{*}}>0$, the optimal cycle length $\hat{t}_{N}^{*}$ is either $t_{N}^{*}$ if $l^{*}=l_{0}$ or $t_{N_{l}}$ if $l^{*}>l_{0}$ and the optimal order quantity is determined by

$$
Q^{*}=\sum_{i=1}^{N} D_{i}^{*}\left(\hat{t}_{N}^{*}\right)\left(t_{i}^{*}\left(\hat{t}_{N}^{*}\right)-t_{i-1}^{*}\left(\hat{t}_{N}^{*}\right)\right) .
$$

Otherwise, it is not optimal to order, i.e., $Q^{*}=0$.

\section{END}

This procedure resembles the algorithm for the classical EOQ average cost minimization problem with an all-units quantity discount. It takes into account the interdependencies of the determination of the optimal order quantity, the optimal prices in the $N$ time intervals, and the optimal times where the price is adjusted. In Step 1 we solve the constrained optimization problems backwards, starting with the highest discount rate $r_{L}$ until an unconstrained solution is feasible for the first time. In Step 2, we compare the various breakpoint profits for $L$ to $l_{0}+1$ to the first free optimal solution and identify the optimal discount rate $r_{l}^{*}$ where the profit reaches the maximum. In Step 3 we determine the resulting optimal cycle length and the optimal order quantity.

\section{Linear price-response function}

In this section, we detail the previous results for the special case of a linear price-response function. We show that for each discount rate $r_{l}$ both the optimal prices and the average profit can be uniquely expressed as a closed-form single-variable function of $t_{N}$, e.g., $P_{i}^{*}\left(t_{N}\right)$ for all $i=1, \cdots, N$ and $\Pi_{l}\left(t_{N}\right)$. The linear price-response function satisfies all conditions of Section 3.1. We will show that $\Pi_{l}\left(t_{N}\right)$ is a concave-convex function of $t_{N}$ and provide a detailed algorithm for determining the overall optimal decisions of the dynamic coordinated selling price strategy and the lot-size. A detailed analysis of the decentralized framework and the coordinated-constant pricing framework for linear price-response functions is given in Eliashberg and Steinberg (1993) and Abad (1988a). Let

$$
D=D(P)= \begin{cases}a-b P & \text { if } 0 \leq P \leq \frac{a}{b} \\ 0 & \text { if } P>\frac{a}{b}\end{cases}
$$

where $a$ represents the market potential and the reservation price is $\bar{P}=\frac{a}{b}$.

Using the results of Transchel and Minner (2005), the optimal times $t_{i}^{*}$ can be represented by functions of $t_{N}$

$$
t_{i}^{*}\left(t_{N}\right)=i \frac{t_{N}}{N}
$$

the optimal prices and demand rates are characterized by

$$
\begin{aligned}
\text { (23) } P_{i}^{*}\left(t_{N}\right) & =\frac{1}{2}\left(\frac{a}{b}+c_{l}+\frac{h_{l}}{2}(2 i-1) \frac{t_{N}}{N}\right) \\
(24) \Rightarrow D_{i}^{*}\left(t_{N}\right) & =\frac{b}{2}\left(\frac{a}{b}-c_{l}-\frac{h_{l}}{2}(2 i-1) \frac{t_{N}}{N}\right)
\end{aligned}
$$

and by inserting (22), (23), and (24) into (18), the profit function for a given purchase price $c_{l}$ can be transformed into the single-variable function

$$
\begin{aligned}
\Pi_{l}^{(N)}\left(t_{N}\right)= & \frac{b}{4}\left[\left(\frac{a}{b}-c_{l}\right)\left(\frac{a}{b}-c_{l}-h_{l} t_{N}\right)\right. \\
& \left.+\frac{h_{l}^{2} t_{N}^{2}}{12} \frac{\left(4 N^{2}-1\right)}{N^{2}}\right]-\frac{F}{t_{N}}
\end{aligned}
$$

For a detailed derivation of (25) see Appendix A.4.

Property 4 For an arbitrary $t_{N},(25)$ is a decreasing function of $c_{l}$. Therefore, the profit functions $\Pi_{l}^{(N)}\left(t_{N}\right)$ and $\Pi_{l^{\prime}}^{(N)}\left(t_{N}\right)$ do not intersect for $c_{l} \neq$ $c_{l}$, i.e., $\Pi_{l}^{(N)}\left(t_{N}\right)>\Pi_{l-1}^{(N)}\left(t_{N}\right)$ for all $l=1, \cdots, N-1$.

Property 5 If $D=D(P)$ is linear, the profit function $\Pi_{l}^{(N)}\left(t_{N}\right)$ is a concave-convex function of $t_{N}$.

The proofs of Property 4 and 5 are given in Appendix A.5 and A.6. With the results of Properties 4 and 5, all requirements to use Algorithm 2 are satisfied.

\section{Algorithm:}

Step (1):

Solve the constrained optimization problem (19)-(20) from $l=L$ until the largest index $l_{0}$ is identified where $\bar{Q}_{l} \leq Q_{l_{0}}^{*}<\bar{Q}_{l_{0}+1}$ by using the Lagrangian Multiplier method. We introduce the Lagrangian multipliers $\lambda_{1}$ and $\lambda_{2}^{i}$ for $i=1, \cdots, N$ related to the minimum order quantity constraint and the reservation price constraints for all charged selling prices over an order cycle. The Lagrangian 
function $\mathcal{L}_{l}$ for a particular purchasing cost $c_{l}$ is defined by:

$$
\begin{aligned}
\mathcal{L}_{l}\left(P_{1}, \cdots, P_{N}, t_{N}, \lambda_{1}, \lambda_{2}^{i}\right) \\
=\frac{1}{t_{N}}\left[\sum_{i=1}^{N}\left(P_{i}-c_{l}-\frac{h_{l}}{2}(2 i-1) \frac{t_{N}}{N}\right)\right. \\
\left.\quad \times\left(a-b P_{i}\right) \frac{t_{N}}{N}-F\right] \\
+\lambda_{1}\left[\frac{t_{N}}{N} \sum_{i=1}^{N}\left(a-b P_{i}\right)-\bar{Q}_{l}\right] \\
+\sum_{i=1}^{N} \lambda_{2}^{i}\left[\frac{a}{b}-P_{i}\right],
\end{aligned}
$$

with the Kuhn-Tucker conditions

$$
\begin{array}{ll}
\frac{\partial \mathcal{L}_{l}}{\partial P_{i}} \leq 0, & P_{i}^{*} \frac{\partial \mathcal{L}_{l}}{\partial P_{i}}=0, \quad \forall i=1, \cdots, N \\
\frac{\partial \mathcal{L}_{l}}{\partial t_{N}} \leq 0, & t_{N}^{*} \frac{\partial \mathcal{L}_{l}}{\partial t_{N}}=0, \\
\frac{\partial \mathcal{L}_{l}}{\partial \lambda_{1}} \geq 0, & \lambda_{1}^{*} \frac{\partial \mathcal{L}_{l}}{\partial \lambda_{1}}=0, \\
\frac{\partial \mathcal{L}_{l}}{\partial \lambda_{2}^{i}} \geq 0, & \lambda_{2}^{i *} \frac{\partial \mathcal{L}_{l}}{\partial \lambda_{2}^{i}}=0, \quad \forall i=1, \cdots, N \\
& \lambda_{1} \geq 0, \lambda_{2}^{i} \geq 0, \quad \forall i=1, \cdots, N .
\end{array}
$$

We seek the price vector $P_{1}^{*}, P_{2}^{*}, \cdots, P_{N}^{*}$ and the cycle length $t_{N}^{*}$ that satisfy all these conditions. The partial derivation with respect to $P_{i}$ for all $i=$ $1, \cdots, N$, and the partial derivation with respect to $t_{N}$ gives

$$
\begin{aligned}
\frac{\partial \mathcal{L}_{l}}{\partial P_{i}}= & \frac{1}{N}\left[a-b P_{i}+\left(P_{i}-c_{l}-\frac{h_{l}}{2}(2 i-1) \frac{t_{N}}{N}\right)(-b)\right. \\
& \left.-\lambda_{1} b t_{N}-\lambda_{2}^{i} N\right] \stackrel{!}{=} 0
\end{aligned}
$$

$$
\begin{aligned}
& \Leftrightarrow P_{i}\left(t_{N}, \lambda_{1}, \lambda_{2}^{i}\right)= \\
& \frac{1}{2}\left(\frac{a}{b}+c_{l}+\frac{h_{l}}{2}(2 i-1) \frac{t_{N}}{N}-\lambda_{1} t_{N}-\lambda_{2}^{i} N\right) .
\end{aligned}
$$

$$
\begin{aligned}
\frac{\partial \mathcal{L}_{l}}{\partial t_{N}}= & \frac{F}{t_{N}^{2}}-\frac{h_{l}}{2}\left(a-\frac{b}{N^{2}} \sum_{i=1}^{N}\left(P_{i}(2 i-1)\right)\right) \\
& +\lambda_{1}\left(a-\frac{b}{N} \sum_{i=1}^{N} P_{i}\right) \stackrel{!}{=} 0
\end{aligned}
$$

(27) $\Leftrightarrow t_{N}\left(P_{1}, \cdots, P_{N}, \lambda_{1}\right)=$

$\sqrt{\frac{2 F}{h_{l}\left(a-\frac{b}{N^{2}} \sum_{i=1}^{N}\left(P_{i}(2 i-1)\right)\right)-2 \lambda_{1}\left(a-\frac{b}{N} \sum_{i=1}^{N} P_{i}\right)}}$.

Subject to the complementary slackness condition, we obtain the first-order condition

$$
\frac{\partial \mathcal{L}_{l}}{\partial \lambda_{1}}=\frac{t_{N}}{N} \sum_{i=1}^{N}\left(a-b P_{i}\right)-\bar{Q}_{l} \stackrel{!}{\geq} 0 .
$$

The two constraints (28) and $\lambda_{1} \geq 0$ are complementary inequalities, i.e., $\frac{\partial \mathcal{L}_{l}}{\partial \lambda_{1}}>0$ if and only if $\lambda_{1}=0$. Thus, if $\lambda_{1}>0$, it must be satisfied that $\frac{\partial \mathcal{L}_{l}}{\partial \lambda_{1}}=0$. The value of $\lambda_{1}$ can be interpreted as shadow price that values a decrease of the breakpoint quantity $\bar{Q}_{l}$ by one unit. Equation (26) indicates that if $h_{l}>0$ the optimal selling price is strictly increasing over the order cycle. Therefore, the reservation price constraints must not be binding for $i=1, \cdots, N-1$, that is, $\lambda_{2}^{i}=0$ for $i=1, \cdots, N-1$. The only reservation price that may constrain the problem is for $P_{N}\left(t_{N}\right) \leq \bar{P}_{N}$ :

$$
\frac{\partial \mathcal{L}_{l}}{\partial \lambda_{2}^{N}}=\frac{a}{b}-P_{N} \stackrel{!}{\geq} 0 .
$$

A similar shadow price interpretation holds for $\lambda_{2}^{N}$. Here, (29) and $\lambda_{2}^{N} \geq 0$ are complementary inequalities, i.e., $\lambda_{2}^{N}>0$ if and only if $P_{N}=\frac{a}{b}$. Substituting $P_{N}$ from (26) into (29) gives an upper bound for the optimal cycle length

$$
t_{N} \leq \frac{\frac{a}{b}-c_{l}+\lambda_{2}^{N} N}{h_{l}\left(\frac{2 N-1}{N}-\lambda_{1}\right)}=t_{N_{l}} .
$$

For determining the optimal solution $\left(P_{1}^{*}, \cdots\right.$, $\left.P_{N}^{*}, t_{N}^{*}, \lambda_{1}^{*}, \lambda_{2}^{N^{*}}\right)$, we solve the system of equations and inequalities (26), (27), (28), and (30) under the condition $\lambda_{1}^{*} \geq 0$ and $\lambda_{2}^{N^{*}} \geq 0$.

Step (2) and Step (3) follow straight just as described in Algorithm 2.

\section{Numerical example}

The following numerical example shows the benefit of coordinated planning of price and order quantity compared to a decentralized planning approach and the impact of the ratio of fixed and holding cost, price sensitivity of customers, and the suppliers quantity discount. The demand follows the linear 
Table 1: Results of the base case (Parameter: $b=100, \bar{Q}_{1}=4000, r_{1}=0.15$ )

\begin{tabular}{|c|c|c|c|c|c|c|c|c|c|c|c|}
\hline \multirow[b]{2}{*}{ Framework } & \multirow{2}{*}{$\begin{array}{l}\text { QD } \\
l\end{array}$} & \multicolumn{5}{|l|}{$\boldsymbol{F}=\mathbf{0}$} & \multicolumn{5}{|c|}{$F=500$} \\
\hline & & $Q_{l}^{*}$ & $T_{l}^{*}$ & $\boldsymbol{P}_{l}^{*}$ & $\Pi_{l}^{*}$ & Loss & $Q_{l}^{*}$ & $T_{l}^{*}$ & $P_{l}^{*}$ & $\Pi_{l}^{*}$ & Loss \\
\hline \multirow{2}{*}{ decentralized } & 0 & 0 & 0.0 & 7.5 & 625 & $0.8 \%$ & 1414 & 5.6 & 7.5 & 448 & \\
\hline & 1 & 4000 & 16.0 & 7.5 & 600 & & 4000 & 16.0 & 7.5 & 568 & $3.9 \%$ \\
\hline \multirow{2}{*}{$\begin{array}{l}\text { coordinated- } \\
\text { constant }\end{array}$} & 0 & 0 & 0.0 & 7.5 & 625 & & 1361 & 5.8 & 7.7 & 451 & \\
\hline & 1 & 4000 & 13.9 & 7.1 & 627 & $0.5 \%$ & 4000 & 14.2 & 7.1 & 588 & $0.5 \%$ \\
\hline \multirow{2}{*}{$\begin{array}{l}\text { coordinated- } \\
\text { dynamic }\end{array}$} & 0 & 0 & 0.0 & 7.5 & 625 & & 1374 & $(2.9,5.9)$ & $(7.6,7.8)$ & 452 & \\
\hline & 1 & 4000 & $(7.0,14.0)$ & $(6.9,7.3)$ & 630 & & 4000 & $(7.1,14.2)$ & $(7.0,7.4)$ & 591 & \\
\hline
\end{tabular}

Table 2: Impact of a decreasing minimum order quantity $Q_{1}$ (Parameter: $b=100$, $\left.\bar{Q}_{1}=3000, r_{1}=0.15\right)$

\begin{tabular}{|c|c|c|c|c|c|c|c|c|c|c|c|}
\hline \multirow[b]{2}{*}{ Framework } & \multirow{2}{*}{$\begin{array}{l}\text { QD } \\
l\end{array}$} & \multicolumn{5}{|c|}{$\boldsymbol{F}=\mathbf{0}$} & \multicolumn{5}{|c|}{$F=500$} \\
\hline & & $Q_{l}^{*}$ & $T_{l}^{*}$ & $\boldsymbol{P}_{l}^{*}$ & $\Pi_{l}^{*}$ & Loss & $Q_{l}^{*}$ & $T_{l}^{*}$ & $\boldsymbol{P}_{l}^{*}$ & $\Pi_{l}^{*}$ & Loss \\
\hline \multirow[t]{2}{*}{ decentral } & 0 & 0 & 0.0 & 7.5 & 625 & & 1414 & 5.6 & 7.5 & 448 & \\
\hline & 1 & 3000 & 12.0 & 7.5 & 653 & $3.4 \%$ & 3000 & 12.0 & 7.5 & 611 & $2.2 \%$ \\
\hline \multirow{2}{*}{$\begin{array}{l}\text { coordinated- } \\
\text { constant }\end{array}$} & 0 & 0 & 0.0 & 7.5 & 625 & & 1361 & 5.8 & 7.7 & 451 & \\
\hline & 1 & 3000 & 10.4 & 7.1 & 674 & $0.3 \%$ & 3000 & 10.7 & 7.2 & 624 & $0.2 \%$ \\
\hline \multirow{2}{*}{$\begin{array}{l}\text { coordinated- } \\
\text { dynamic }\end{array}$} & 0 & 0 & 0.0 & $(7.5,7.5)$ & 625 & & 1374 & $(2.9,5.9)$ & $(7.6,7.7)$ & 452 & \\
\hline & 1 & 3000 & $(5.2,10.5)$ & $(6.9,7.3)$ & 676 & & 3000 & $(5.3,10.7)$ & $(7.0,7.4)$ & 625 & \\
\hline
\end{tabular}

structure $D(P)=a-b P$. The parameters are set to $a:=1000, c_{0}:=5$, and $h_{l}:=0.02 c_{l}$. The parameters $F, \bar{Q}_{1}, r_{1}$, and $b$ are varied in order to show the impact on lot-sizing and pricing. In the case of a dynamic pricing strategy, we assume that the retailer changes the selling price twice in each order cycle, i.e., $N=2$.

As a base case, we consider that the price sensitivity factor is $b=100$ and the supplier discount is $r_{1}=15 \%$ if $Q \geq 4000=: \bar{Q}_{1}$ units. Furthermore, we distinguish $F=0$ and $F=500$. Due to the fact that purchasing price and interest rate are fixed, $F=0$ represents the case of a low and $F=500$ the case of a high fixed-holding cost ratio. Table 1 shows the optimal price and lot-sizing decision as well as the optimal cycle length and the optimal average profit for the three frameworks and both purchasing options regular $(l=0)$ and discounted $(l=1)$. Column "Loss" illustrates the loss of profit compared to the coordinated-dynamic framework. If $F=500$ and the retailer does not use the quantity discount $(l=0)$, we verify the findings of Eliashberg and Steinberg (1993) that decentralized decision making leads to an underestimation of price and an overestimation of lot-size compared to coordinated decision making $(7.5<7.7$ and $1414>1361)$. However, this is not neces- sarily true if the supplier offers a quantity discount. If the retailer uses the quantity discount $(l=1)$, for both $F=0$ and $F=500$ it can be observed that the optimal selling price decreases between the decentralized and coordinated-constant framework $(7.5>7.1)$ whereas the order-quantity is equal. In case of decentralized decision and $F=0$, the optimal purchasing strategy is a just-intime strategy with the regular purchasing cost $c_{0}$ $\left(\Pi_{0}^{*}=625>600=\Pi_{1}^{*}\right)$ and an optimal selling price $P_{0}^{*}=7.5$. However, if the retailer optimizes price and purchasing policy simultaneously, the discount strategy becomes more profitable. That is, the discount effect dominates the coordination effect (see Section 3). It is optimal to accept additional holding costs in order to save variable purchasing costs. Due to the larger lot-size which would result in higher holding costs, it becomes more beneficial to decrease the selling pricing in order to increase the demand rate. In case of $F=500$, the lot-sizing decision is equal for the decentralized and coordinated framework. However, in the coordinated framework a lower selling price leads to a higher order frequency (a higher demand rate leads to a lower cycle length) which results in reduced holding cost. Furthermore, it can be seen that the benefit of dynamic pricing 
Table 3: Impact of a increasing discount rate $r_{1}$ (Parameter: $b=100, \bar{Q}_{1}=4000$, $\boldsymbol{r}_{1}=\mathbf{0 . 2 )}$

\begin{tabular}{|c|c|c|c|c|c|c|c|c|c|c|c|}
\hline \multirow[b]{2}{*}{ Framework } & \multirow{2}{*}{$\begin{array}{l}\text { QD } \\
l\end{array}$} & \multicolumn{5}{|l|}{$F=0$} & \multicolumn{5}{|c|}{$F=500$} \\
\hline & & $Q_{l}^{*}$ & $T_{l}^{*}$ & $\boldsymbol{P}_{l}^{*}$ & $\Pi_{l}^{*}$ & Loss & $Q_{l}^{*}$ & $T_{l}^{*}$ & $P_{l}^{*}$ & $\Pi_{l}^{*}$ & Loss \\
\hline \multirow[t]{2}{*}{ decentral } & 0 & 0 & 0.0 & 7.5 & 625 & & 1414 & 5.6 & 7.5 & 448 & \\
\hline & 1 & 4000 & 16.0 & 7.5 & 670 & $6.0 \%$ & 4000 & 16.0 & 7.5 & 643 & $4.5 \%$ \\
\hline \multirow{2}{*}{$\begin{array}{l}\text { coordinated- } \\
\text { constant }\end{array}$} & 0 & 0 & 0.0 & 7.5 & 625 & & 1361 & 5.8 & 7.7 & 451 & \\
\hline & 1 & 4000 & 13.3 & 7.0 & 711 & $0.3 \%$ & 4000 & 13.6 & 7.0 & 670 & $0.4 \%$ \\
\hline \multirow{2}{*}{$\begin{array}{l}\text { coordinated- } \\
\text { dynamic }\end{array}$} & 0 & 0 & 0.0 & $(7.5,7.5)$ & 625 & & 1374 & $(2.9,5.9)$ & $(7.6,7.7)$ & 452 & \\
\hline & 1 & 4000 & $(7.6,13.4)$ & $(6.9,7.1)$ & 713 & & 4000 & $(6.8,13.6)$ & $(6.9,7.3)$ & 673 & \\
\hline
\end{tabular}

Table 4: Impact of an increasing price sensitivity of customers $b$ (Parameter: $b=140$, $\left.\bar{Q}_{1}=4000, r_{1}=0.15\right)$

\begin{tabular}{|c|c|c|c|c|c|c|c|c|c|c|c|}
\hline \multirow[b]{2}{*}{ Framework } & \multirow{2}{*}{$\begin{array}{l}\text { QD } \\
l\end{array}$} & \multicolumn{5}{|l|}{$F=\mathbf{0}$} & \multicolumn{5}{|c|}{$F=500$} \\
\hline & & $Q_{l}^{*}$ & $T_{l}^{*}$ & $\boldsymbol{P}_{l}^{*}$ & $\Pi_{l}^{*}$ & Loss & $Q_{l}^{*}$ & $T_{l}^{*}$ & $\boldsymbol{P}_{l}^{*}$ & $\Pi_{l}^{*}$ & Loss \\
\hline \multirow[t]{2}{*}{ decentral } & 0 & 0 & 0.0 & 6.0 & 161 & $0.0 \%$ & 1095 & 7.3 & 6.0 & 23 & \\
\hline & 1 & 4000 & 26.0 & 6.0 & 61 & & 4000 & 26.0 & 6.0 & 42 & $55.8 \%$ \\
\hline \multirow{2}{*}{$\begin{array}{l}\text { coordinated- } \\
\text { constant }\end{array}$} & 0 & 0 & 0.0 & 6.0 & 161 & $0.0 \%$ & 951 & 8.4 & 6.3 & 32 & \\
\hline & 1 & 4000 & 19.0 & 5.7 & 118 & & 4000 & 20.0 & 5.7 & 88 & $7.4 \%$ \\
\hline \multirow{2}{*}{$\begin{array}{l}\text { coordinated- } \\
\text { dynamic }\end{array}$} & 0 & 0 & 0.0 & $(6.0,6.0)$ & 161 & & 987 & $(4.5,9.0)$ & $(6.2,6.5)$ & 35 & \\
\hline & 1 & 4000 & $(10.0,20.0)$ & $(5.5,6.0)$ & 124 & & 4000 & $(11.0,21.0)$ & $(5.5,6.1)$ & 95 & \\
\hline
\end{tabular}

compared to coordinated constant pricing for both $F=0$ and $F=500$ is lower than $1 \%$. The same observation can be made when we compare the dynamic-coordinated framework with the decentralized framework for the case $F=0$. If $F=0$, the benefit of coordinated-dynamic pricing increases to $4 \%$ per time unit. Tables 2 and 3 illustrate the impact of the discount policy on the optimal solution. Table 2 shows the results of a decreasing minimum order quantity and Table 3 the results of an increasing discount rate. Both changes yield an increasing desirability of the quantity discount. While the benefit of the coordinated-dynamic framework compared to the coordinated-constant framework remains low, the benefit compared to the decentralized framework does increase. Compared to the base case $F=0$ where a JIT policy was optimal, now the quantity discount is beneficial such that the retailer orders the minimum order quantity. Table 4 illustrates the impact of price sensitivity on the optimal decision and the average profit. Compared to the base case, an increasing price sensitivity leads to a decreasing selling price among all decision frameworks. For $F=0$, across all decision frameworks, it is optimal to follow a JIT strategy. Therefore, decentralized decision making yields the same performance as coordinated decision making. But, in case of $F=500$, the application of coordinated-dynamic pricing is highly beneficial compared to decentralized and coordinated-constant decision making.

\section{Conclusion}

This paper considered an economic order quantity (EOQ) model when the supplier offers an allunits quantity discount (AQD) and the retailer faces a price-dependent demand rate. We analyzed three different decision frameworks, a decentralized decision-making strategy, a coordinated strategy with a constant selling price, and a coordinated strategy where the retailer is allowed to implement a finite number of price adjustments within each order cycle. For the coordinated frameworks, we derived analytical properties used to develop efficient algorithms in order to determine the optimal price and lot-sizing strategy. These algorithms are applicable to all concave, quasiconcave, and concave-convex profit functions. In a numerical example we showed the difference in decision making between the decentralized, the coordinated-constant, and the coordinated- 
dynamic framework. It could be observed that in the case of low price sensitivity of the customers, the benefit of dynamic pricing compared to constant pricing is rather low. However, with increasing price sensitivity, coordinated decision making and in particular dynamic pricing becomes more beneficial.

Without a supplier quantity discount, the coordinated framework with a constant pricing strategy yields a higher price, a lower demand rate, and a lower order quantity compared to sequential pricing and replenishment (see Eliashberg and Steinberg (1993)). If the supplier offers an allunits quantity discount, these properties do not necessarily hold. In the discount case, two effects influence the outcome of decision making. The first effect is the overhead cost effect. In contrast to decentralized decision-making, a central decision maker takes into account all relevant costs (setup cost and inventory holding cost). This yields an increasing selling price, a decreasing demand rate, and a decreasing lot-size. The second effect is the discount effect. Here, under decentralized decision making, marketing does not consider the quantity discount schedule of the supplier and optimizes the selling price only using the undiscounted purchasing price. Thus, marketing overestimates the cost which yields a higher selling price than in the case of coordinated decision making. However, by taking into account the supplier quantity discount it might be optimal to order a larger lot-size at a reduced purchasing price. Therefore, an increase or decrease of the selling price, the demand rate, and the order quantity depends on which effect dominates, the overhead cost effect that yields an underestimation of the average cost in the decentralized system, or the quantity discount effect where the decentralized system overestimates the average cost.

A weakness of the prescribed model is the deterministic and stationary environment based on the EOQ assumptions. Furthermore, we disregard strategic behavior of customers. Additional research is needed to analyze the problem where customers are willing to buy earlier (forward buying) or later (postponement) if prices vary over time. A natural extension of the model would be to consider a joint dynamic pricing and lot-sizing determination problem where the supplier offers an incremental units discount. An extension regarding supply chain coordination is to investigate the problem, how does the supplier design a quantity discount schedule if it is anticipated that the retailer employs decentralized decision making, coordinated decision making with a constant price, or coordinated decision making with a dynamic pricing framework.

\section{A Appendix}

\section{A.1 Proof of Property 1}

Let $C(P):=\sqrt{2 F h_{l} D(P)} . C(P)$ is a monotonically decreasing function in $P$, i.e., $\frac{\partial C}{\partial P} \leq 0$. Furthermore, $\frac{\partial^{2} C}{\partial P^{2}}=\sqrt{\frac{F h_{l}}{2 D(P)}}\left(\frac{\left(D^{\prime}(P)\right)^{2}}{2 D(P)}-D^{\prime \prime}(P)\right)$. If $D^{\prime \prime}(P) \leq 0$, then $C(P)$ is a convex function in $P$, i.e., $\frac{\partial C}{\partial P} \geq 0$. Therefore, $-C(P)$ is a concave function in $P$. Because $\tilde{\Pi}_{l}$ is a concave function in $P, \Pi_{l}(P)$ is a concave function. However, if $D^{\prime \prime}(P)>0$, then $C(P)$ is not uniquely convex or concave. For all $P$ with $\frac{\left(D^{\prime}(P)\right)^{2}}{2 D(P)}-D^{\prime \prime}(P) \geq 0, C(P)$ is convex and it follows the same argumentation as above. For all $P$ where $\frac{\left(D^{\prime}(P)\right)^{2}}{2 D(P)}-D^{\prime \prime}(P) \leq 0, C(P)$ is concave and thus, $-C(P)$ is convex. Due to the monotonicity, $\Pi_{l}(P)$ is concave-convex. An analysis of the limiting values gives $\lim _{P \rightarrow 0} \Pi_{l}(P)=-\sqrt{2 F h_{l} D(0)} \leq 0$ and $\lim _{P \rightarrow \bar{P}} \Pi_{l}(P)=0$.

\section{A.2 Proof of Property 3}

Given the first-order conditions in (9), it follows that we have to show that $\frac{\partial}{\partial P}\left(\frac{D(P)}{D^{\prime}(P)}+P\right) \geq 0$. The optimal $P$ has to satisfy $\frac{D(P)}{D^{\prime}(P)}+P \geq 0 \Leftrightarrow \varepsilon=$ $-\frac{D^{\prime}(P)}{D(P)} P \geq 1$. The IPE from Definition 1 gives that

$$
\begin{aligned}
\frac{\partial \varepsilon}{\partial P}=-\frac{\partial}{\partial P}\left(\frac{D^{\prime}(P)}{D(P)} P\right) & \geq 0 \\
\Leftrightarrow-\frac{\partial}{\partial P}\left(\frac{D^{\prime}(P)}{D(P)}\right) P-\frac{D^{\prime}(P)}{D(P)} & \geq 0 .
\end{aligned}
$$

Some algebraic transformations give

(31) $-\frac{D^{\prime \prime}(P)}{D^{\prime}(P)} P \leq 1+\varepsilon$.

Assume that $\frac{\partial}{\partial P}\left(\frac{D(P)}{D^{\prime}(P)}+P\right)<0$. Thus $\frac{\partial}{\partial P}\left(\frac{D(P)}{D^{\prime}(P)}\right)+$ $1<0 \Leftrightarrow 2-\frac{D(P) D^{\prime \prime}(P)}{\left(D^{\prime}(P)\right)^{2}}<0$. Then,

(32) $-\frac{D^{\prime \prime}(P)}{D^{\prime}(P)} P>2 \varepsilon$.

Because $\varepsilon \geq 1$, inequality (31) and (32) can not be valid simultaneously. Thus, $\frac{\partial}{\partial P}\left(\frac{D(P)}{D^{\prime}(P)}+P\right) \geq 0$. 
From $c_{0}<c_{0}+\frac{F}{Q_{0}^{*}}$ and (9) we get that $\tilde{P}^{*}<P^{*}$. However, it is not assured that $c_{0}<c_{l}+\frac{F}{Q_{l}^{*}}$ for a quantity discount $r_{l}$ with $l>0$. From the fact that $\frac{\partial}{\partial P}\left(\frac{D(P)}{D^{\prime}(P)}+P\right) \geq 0$, it follows that the selling price in the coordinated system is higher if and only if

$$
c_{0}<c_{l}+\frac{F}{Q}
$$

with $Q=\sqrt{\frac{2 F D(P)}{h_{l}}}$. The property follows from

$$
\begin{aligned}
& c_{0}-c_{l}<\sqrt{\frac{F h_{l}}{2 D(P)}} \Leftrightarrow D(P)<\frac{F h_{l}}{2\left(c_{0}-c_{l}\right)^{2}} \\
& \Leftrightarrow P_{l}^{*}>D^{-1}\left(\frac{F h_{l}}{2\left(c_{0}-c_{l}\right)^{2}}\right) .
\end{aligned}
$$

\section{A.3 Derivation of equation (15)}

An expansion of the term

$$
\left(\sum_{i=1}^{N-1}\left(\left(t_{i}-t_{i-1}\right) \sum_{j=i+1}^{N} D_{j}\left(t_{j}-t_{j-1}\right)\right)\right)
$$

from equation (11) gives

$$
\begin{aligned}
& \left(\sum_{i=1}^{N-1} t_{i} \sum_{j=i+1}^{N} D_{j}\left(t_{j}-t_{j-1}\right)\right) \\
& -\left(\sum_{i=1}^{N-1} t_{i-1} \sum_{j=i+1}^{N} D_{j}\left(t_{j}-t_{j-1}\right)\right) .
\end{aligned}
$$

With $t_{0}=0$, we can renumber the indices

$$
\begin{aligned}
& \left(\sum_{i=1}^{N-1} t_{i} \sum_{j=i+1}^{N} D_{j}\left(t_{j}-t_{j-1}\right)\right) \\
& \quad-\left(\sum_{i=1}^{N-1} t_{i} \sum_{j=i+2}^{N} D_{j}\left(t_{j}-t_{j-1}\right)\right) \\
& =\sum_{i=1}^{N-1} t_{i} D_{i+1}\left(t_{i+1}-t_{i}\right) \\
& =\sum_{i=1}^{N} t_{i-1} D_{i}\left(t_{i}-t_{i-1}\right) .
\end{aligned}
$$

Therefore, (11) can be modified to

$$
\begin{aligned}
\Pi_{l}^{(N)}= & \frac{1}{t_{N}}\left[\sum_{i=1}^{N}\left(P_{i}-c_{l}\right) D_{i}\left(t_{i}-t_{i-1}\right)\right. \\
& -\frac{h_{l}}{2} \sum_{i=1}^{N} D_{i}\left(t_{i}-t_{i-1}\right)^{2} \\
& \left.-h_{l} \sum_{i=1}^{N} D_{i} t_{i-1}\left(t_{i}-t_{i-1}\right)-F\right] \\
= & \frac{1}{t_{N}}\left[\sum_{i=1}^{N}\left(P_{i}-c_{l}-\frac{h_{l}}{2}\left(t_{i}+t_{i-1}\right)\right)\right. \\
& \left.\times D_{i}\left(t_{i}-t_{i-1}\right)-F\right] .
\end{aligned}
$$

\section{A.4 Derivation of (25)}

From (18) and (22)-(24) follows

$$
\begin{aligned}
\Pi_{l}^{(N)}= & \frac{1}{t_{N}} \sum_{i=1}^{N}\left[\left(\frac{1}{2}\left(\frac{a}{b}-c_{l}+\frac{h_{l}}{2}(2 i-1) \frac{t_{N}}{N}\right)\right.\right. \\
& \left.-\frac{h_{l}}{2}(2 i-1) \frac{t_{N}}{N}\right) \\
& \left.\times \frac{b}{2}\left(\frac{a}{b}-c_{l}-\frac{h_{l}}{2}(2 i-1) \frac{t_{N}}{N}\right) \frac{t_{N}}{N}\right]-\frac{F}{t_{N}} \\
= & \frac{1}{t_{N}} \sum_{i=1}^{N}\left[\frac { b } { 4 } \frac { t _ { N } } { N } \sum _ { i = 1 } ^ { N } \left(\frac{a}{b}-c_{l}\right.\right. \\
& \left.\left.-\frac{h_{l}}{2}(2 i-1) \frac{t_{N}}{N}\right)^{2}\right]-\frac{F}{t_{N}} \\
= & \frac{1}{t_{N}} \sum_{i=1}^{N}\left[\frac { b } { 4 } \frac { t _ { N } } { N } \left[\left(\frac{a}{b}-c_{l}\right)^{2} N-\left(\frac{a}{b}-c_{l}\right) h_{l} t_{N} N\right.\right. \\
& \left.\left.+\frac{h_{l}^{2}}{4} \frac{t_{N}^{2}}{N^{2}} \sum_{i=1}^{N}(2 i-1)^{2}\right]\right]-\frac{F}{t_{N}} .
\end{aligned}
$$

Therefore,

$$
\begin{aligned}
\Pi_{l}^{(N)}\left(t_{N}\right)= & \frac{b}{4}\left[\left(\frac{a}{b}-c_{l}\right)\left(\frac{a}{b}-c_{l}-h_{l} t_{N}\right)\right. \\
& \left.+\frac{h_{l}^{2} t_{N}^{2}}{12} \frac{\left(4 N^{2}-1\right)}{N^{2}}\right]-\frac{F}{t_{N}} .
\end{aligned}
$$




\section{A.5 Proof of Property 4}

The partial derivation of (25) gives

$$
\begin{aligned}
\frac{\partial \Pi_{l}^{(N)}}{\partial c_{l}}= & -\left(\frac{a}{b}-c_{l}-h_{l} t_{N}\right) \\
& -\left(\frac{a}{b}-c_{l}\right)\left(1+\frac{\partial h_{l}}{\partial c_{l}} t_{N}\right) \\
& +\frac{h_{l}}{6} \frac{\partial h_{l}}{\partial c_{l}} \frac{\left(4 N^{2}-1\right)}{N^{2}} t_{N}^{2} \\
= & -2\left(\frac{a}{b}-c_{l}-\frac{h_{l}}{2} t_{N}\right) \\
& -\left(\frac{a}{b}-c_{l}\right) \frac{\partial h_{l}}{\partial c_{l}} t_{N}+\frac{h_{l}}{6} \frac{\partial h_{l}}{\partial c_{l}} \frac{\left(4 N^{2}-1\right)}{N^{2}} t_{N}^{2} \\
= & -2\left(\frac{a}{b}-c_{l}-\frac{h_{l}}{2} t_{N}\right) \\
& -\frac{\partial h_{l}}{\partial c_{l}}\left(\frac{a}{b}-c_{l}-\frac{h_{l}}{6} \frac{\left(4 N^{2}-1\right)}{N^{2}} t_{N}\right) t_{N} .
\end{aligned}
$$

Using $\frac{\partial h_{l}}{\partial c_{l}} \geq 0$ and $D_{i} \geq 0$ for all $i=1, \cdots, N$ with

$$
\begin{aligned}
& D_{N}=\frac{b}{2}\left(\frac{a}{b}-c_{l}-\frac{h_{l}}{2} \frac{(2 i-1)}{N} t_{N}\right) \geq 0 \\
& \Leftrightarrow \frac{a}{b}-c_{l}-\frac{h_{l}}{2} \frac{(2 i-1)}{N} t_{N} \geq 0,
\end{aligned}
$$

it follows

$$
\begin{aligned}
& \frac{\partial \Pi_{l}^{(N)}}{\partial c_{l}}=-2 \underbrace{\left(\frac{a}{b}-c_{l}-\frac{h_{l}}{2} t_{N}\right)}_{\geq 0} \\
&-\frac{\partial h_{l}}{\partial c_{l}} \underbrace{\left(\frac{a}{b}-c_{l}-\frac{h_{l}}{6} \frac{\left(4 N^{2}-1\right)}{N^{2}} t_{N}\right)}_{\geq 0} t_{N} \leq 0 .
\end{aligned}
$$

\section{A.6 Proof of Property 5}

The first-order condition gives that $\Pi_{l}^{(N)}\left(t_{N}\right)$ has three local extreme values. The limiting values indicate that $\lim _{t_{N} \rightarrow-\infty} \Pi_{l}^{(N)}\left(t_{N}\right)=+\infty, \lim _{t_{N} \rightarrow 0^{-}} \Pi_{l}^{(N)}\left(t_{N}\right)=$ $+\infty$. Thus, the function $\Pi_{l}^{(N)}\left(t_{N}\right)$ has a local minimum for $t_{N}<0$ and a vertical asymptote at $t_{N}=0$. Furthermore, we have $\lim _{t_{N} \rightarrow+0} \Pi_{l}^{(N)}\left(t_{N}\right)=-\infty$ and $\lim _{t_{N} \rightarrow+\infty} \Pi_{l}^{(N)}\left(t_{N}\right)=+\infty$. Therefore, for $t_{N} \geq 0$ the profit function $\Pi_{l}^{(N)}\left(t_{N}\right)$ comes from $-\infty$, has first a local maximum, then a local minimum, and goes to $+\infty$, i.e., $\Pi_{l}^{(N)}\left(t_{N}\right)$ is a concave-convex function for $t_{N} \geq 0$.

\section{References}

Abad, Prakash L. (1988a): Determining optimal selling price and lot size when the supplier offers all-unit quantity discounts, Decision Sciences, 19 (3): 622-634.

Abad, Prakash L. (1988b): Joint price and lot-size determination when supplier offers incremental quantity discounts, Journal of the Operational Research Society, 39 (6): 603-607.

Abad, Prakash L. (1997): Optimal policy for a reseller when the supplier offers a temporary reduction in price, Decision Sciences, 28 (3): 637-653.

Benton, W.C. and Seungwook Park (1996): A classification of literature on determining the lot size under quantity discounts, European Journal of Operational Research, 92 (2): 219-238.

Beranek, William (1967): Financial implications of lot-size inventory models, Management Science, 13 (8): 401-408.

Bitran, Gabriel R. and René Caldentey (2003): An overview of pricing models for revenue management, Manufacturing \& Service Operations Management, 5 (3): 203-229.

Burwell, Timothy H., Dinesh S. Dave, Kathy E. Fitzpatrick, and Melvin R. Roy (1991): An inventory model with planned shortages and price dependent demand, Decision Sciences, 22 (5): 1187-1191.

Chan, Lap M. A., Zuo J. M. Shen, David Simchi-Levi, and Julie Swann (2004): Coordination of pricing and inventory decisions: A survey and classification, David Simchi-Levi, S. David Wu, and Zuo J. M. Shen, eds., Handbook of Quantitative Supply Chain Analysis: Modeling in the E-Business Era, Kluwer Academic Publishers, Boston et al., Chapter 9, 335-392.

Dolan, Robert J. (1987): Quantity discount: managerial issues and research opportunities, Marketing Science, 6 (1): 1-22.

Eliashberg, Jehoshua and Richard Steinberg (1993): Marketing-production joint decision making, Jehoshua Eliashberg and Gary L. Lilien, eds., Marketing, Handbooks of Operations Research and Management Science, vol. 5, North Holland, Amsterdam et al., Chapter 18, 827-880.

Elmaghraby, Wedad and Pinar Keskinocak (2003): Dynamic pricing in the presence of inventory considerations: research overview, current practice, and future directions, Management Science, 49 (10): 1287-1309.

Fleischmann, Moritz, Joseph M. Hall, and DavidF. Pyke (2004): Smart pricing: Linking pricing decisions with operational insights, MIT Sloan Management Review, 45 (2): 9-13.

Ghose, Sanjoy and Samar Mukhopadhyay (1993): Quality as an interface between manufacturing and marketing: a conceptual model and an empirical study, Management International Review, 33 (1): 39-52.

Goyal, Suresh K. and Omprakash K. Gupta (1990): A simple approach to the discount purchase problem, Journal of the Operational Research Society, 41 (12): 1169-1170.

Gupta, Omprakash K. (1988): An improved procedure for economic order quantity with all-units price discount, International Journal of Operations \& Production Management, 8 (4): 79-83

Hadley, George and Thomson M. Whitin (1963): Analysis of inventory systems, Prentice-Hall, Englewood Cliffs, New Jersey.

Munson, Charles L. and Meir J. Rosenblatt (1998): Theories and realities of quantity discounts: An exploratory study, Production and Operations Management, 7 (4): 352-369.

Neslin, Scott A., Stephen G. Powell, and Linda S. Stone (1995): The effects of retailer and consumer response on optimal manufacturer advertising and trade promotion strategies, Management Science, 41 (5): 749-766. 
Netessine, Serguei (2006): Dynamic pricing of inventory/capacity with infrequent price changes, European Journal of Operational Research, 174 (1): 553-580.

Rajan, Arvind, Rakesh, and Richard Steinberg (1992): Dynamic pricing and ordering decisions by a monopolist, Management Science, 38 (2): 240-262.

Rubin, Paul A. and W. C. Benton (2003): A generalized framework for quantity discount pricing schedules, Decision Sciences, 34 (1): 173-188.

Transchel, Sandra and Stefan Minner (2005): The impact of dynamic pricing on the economic order decision, Working paper no. 2/2005, University of Mannheim, Available at http://minner.bwl.uni-mannheim.de/hp/_files/ forschung/reports/tr-2005-02.pdf.

Wang, Qinan (2005): Discount pricing policies and the coordination of decentralized distribution systems, Decision Sciences, 36 (4): 627-646.

Weng, Z. Kevin (1995): Channel coordination and quantity discount, Management Science, 41 (9): 1509-1522.

Whitin, Thomson M. (1955): Inventory control and price theory, Management Science, 2 (1): 61-68.

Wilcox, James B., Roy D. Howell, Paul Kuzdrall, and Robert Britney (1987): Price quantity discounts: Some implications for buyers and sellers, Journal of Marketing, 51 (3): 60-70.

\section{Biographies}

Sandra Transchel is a doctoral candidate at the Department of Business Administration and Logistics at the University of Mannheim. She studied Business Mathematics at Otto-von-Guericke University, Magdeburg. Her current research interests include inventory theory and revenue management, with a special focus on dynamic pricing.

Stefan Minner is Professor of Logistics and Supply Chain Management at the University of Vienna. From 2004 to 2008 he held the Chair of Business Administration and Logistics at the University of Mannheim. He studied Business Administration at the University of Bielefeld, received a doctoral degree in 1999 and his habilitation in 2003, both at the University of Magdeburg, and temporarily held the Chair of Operations Management at the University of Paderborn in 2003. His research interests are the design of global logistics networks, inventory management, and the development and application of operations research methods in these fields. Since 2008 Stefan Minner has been Managing Editor of OR Spectrum. 